\title{
The Modified Burr III G family of Distributions
}

\author{
Shahzadi Arifa*, Mohammad Zafar Yab , Azeem Ali \\ National College of Business Administration and Economics, Lahore, Pakistan.
}

\begin{abstract}
We introduce a new family of distributions based on a generalized Burr III generator called Modified Burr III G family and study some of its mathematical properties. Its density function can be bell-shaped, left-skewed, right-skewed, bathtub, J or reversed-J. Its hazard rate can be increasing or decreasing, bathtub, upside-down bathtub, J and reversed-J. Some of its special models are presented. We illustrate the importance of the family with two applications to real data sets.
\end{abstract}

Key words: Burr family of distributions, generalized family, hazard rate, maximum likelihood estimation.

\section{Introduction}

Burr (1942) developed a system of distributions for fitting cumulative frequency distributions. The system includes twelve types of cumulative distribution functions which accommodate a variety of density shapes. The attractiveness of this family of distributions for model fitting is that it combines a simple mathematical expression for the cumulative frequency function with wide coverage in the skewness-kurtosis plane. Many standard distributions, including the Weibull, exponential, logistic, generalized logistic, Gompertz, normal, extreme value and uniform distributions are special cases or limiting cases of the Burr system of distributions.

Among the twelve distribution functions Burr type XII (BXII) and its inverse, type III (BIII) have gathered special attention in physics, actuarial studies, reliability and applied statistics. Nadarajah and Kotz (2007) applied it to fracture toughness and fracture stress data. Gove et al. (2008) fitted Burr III to data related to forestry. Mielke (1973) proposed it as a model for precipitation amounts in the meteorological data. Shao et al. (2008) proposed and fitted an extended Burr III distribution in low-flow frequency analysis where its lower tail was of main interest.

During the last couple of decades new families of probability distributions have been defined to extend well-known families of distributions. The purpose behind is to provide greater flexibility in modeling practical data or the physical or statistical need to explain the mechanism of the generated data. One such approach was by Marshall and Olkin (1997) in which one parameter was added to the survival function of a baseline distribution. Later on Gupta et al. (1998), Gupta and Kundu (1999; 2001a; 2001b; 2002; 2003), Nadarajah and Kotz (2006) and Nadarajah (2011) to name a few who used this approach to generalize their distributions. In another generalization, pioneered by Eugene et al. (2002) and Jones (2004) the beta-generated class from the logit of the beta distribution was defined. Cordeiro and de Castro (2011), Cordeiro 
et al. (2012) used the beta-generated class approach. Kumar (2016) derived new expressions for ratio and inverse moments of lower generalized order-statistics for the Marshall-Olkin extended Burr type xii distribution. Yousof et al.(2016) introduced a new Burr X-G family of distributions by adding a shape parameter.

Gurvich et al. (1997) pioneered a class of extended Weibull distributions which has achieved a prominent position in lifetime models. Its cdf is defined by

$$
F(x ; \alpha, \xi)=1-\exp (-\alpha H(x, \xi)), \quad x \in \square, \alpha>0,
$$

where $\mathrm{H}(\mathrm{x} ; \xi)$ is a non-negative monotonically increasing function which depends on the parameter vector $\xi$. The corresponding pdf is given by

$$
f(x ; \alpha, \xi)=\alpha \exp (-\alpha H(x, \xi)) h(x, \xi),
$$

where $\mathrm{h}(\mathrm{x} ; \xi)$ is the derivative of $\mathrm{H}(\mathrm{x} ; \xi)$.

In this class of distributions $\mathrm{H}(\mathrm{x} ; \xi)$ is taken to be the odds ratio of the baseline distribution.

Another important class of univariate distributions were developed by Zografos and Balakrishnan (2009). They extended Stacy's generalized gamma distribution by replacing $\mathrm{x}$ with $-\log [1-\mathrm{G}(\mathrm{x})]$ and defined their family of distributions having cdf

$$
F(x ; \delta)=\gamma\{\delta,-\log [1-G(x)]\}, \quad x \in \square, \delta>0
$$

with pdf given by

$$
f(x ; \delta)=\frac{1}{\Gamma(\delta)}\{-\log (1-G(x))\}^{\delta-1} g(x)
$$

where

$$
\gamma(\delta, z)=\int_{0}^{z} t^{\delta-1} e^{-t} d t / \Gamma(\delta)
$$

function.

Ristic and Balakrishnan (2012) defined a different type of Gamma-G family of distribution with pdf given by

$$
g(x ; \delta)=\frac{1}{\Gamma(\delta)}(-\log F(x))^{\delta-1} f(x) x \in \square, \delta>0
$$

where $\mathrm{G}(x)$ is any baseline cdf and $\mathrm{g}(\mathrm{x})=\mathrm{dG}(x)$.

Alzaatreh et al. (2013a) proposed a transformed-transformer (T-X) family of distributions. Under this family Gamma-Pareto, Weibull-Pareto and Gamma-Normal distributions were developed by Alzaatreh et al. (2013b), (2012) and (2014) respectively.

In a recent work. Ali et al. (2014) defined Modified Burr III as

$$
f(x)=\alpha \beta x^{-\beta-1}\left[1+\gamma x^{-\beta}\right]^{-\frac{\alpha}{\gamma}-1}, \quad x>0
$$


with distribution function given by

$$
F(x)=\left[1+\gamma x^{-\beta}\right]^{-\frac{\alpha}{\gamma}}
$$

where $\alpha, \beta, \gamma>0$ are shape parameters of the Modified Burr III distribution.

Bourguignon et al. (2014) defined Weibull-G, Nascimento et al. (2014) proposed Gamma extended Weibull family, Santos-Neto et al. (2014) introduced Marshall-Olkin extended Weibull family, Alizadeh et al. (2015) defined Kumaraswamy odd log-logistic family, Tahir et al. (2015a) proposed a new Weibull-G family and Tahir et al. (2015b) introduced the odd generalized exponential family of distributions. Korkmaz and Genç (2015) introduced a generalization of Weibull distribution based on transformation of the two-sided Power distributed random variate. Korkmaz and Genç (2016)studied in detail Two-sided generalized Normal distribution. Maiti et al (2015) defined an Odds Exponential- Exponential distribution for modeling lifetime models. Afify et al. (2016) introduced as Kumaraswamy Transmuted-G Family of distributions, by replacing $\mathrm{x}$ of the cdf of Kumaraswamy distribution with the cdf of the Transmuted Class of distributions.

In this paper, we introduce and study in generality a family of Modified Burr III G distribution, using the Modified Burr III generator applied to the odds ratio of a baseline distribution. By the term "generator" we mean that for each baseline distribution $\mathrm{G}(\mathrm{x})$ we have a different distribution. The objective of writing this paper is to study a new family of distributions which is flexible because of its increasing, decreasing, bathtub, upside-down bathtub, J, reversed$\mathbf{J}$ shaped hazard rate function. With so many practical situations arising in different fields of applications one of these models may provide better fit in a specific practical situation. We also derive some of its mathematical properties and illustrate its usefulness as a better model.

This paper is outlined as follows. In Section 2, we introduce the Modified Burr III G (MBIII G) family of distributions and in Section 3 some special models generated by MBIII G family are given. Section 4 relates to some general mathematical properties. In Section 5 estimation of parameters is performed by the method of maximum likelihood. Section 6 illustrates two applications based on real data sets. Finally concluding remarks are presented in Section7.

\section{Modified Burr III G (MBIII G) Family of Distributions}

Consider a continuous distribution $\mathrm{G}(\mathrm{x} ; \xi)$ with density $\mathrm{g}(\mathrm{x} ; \xi)$ and the cdf of Modified Burr III, given in (1.7). Based on this density we replace $\mathrm{x}$ with the odds ratio $H(x ; \xi)=G(x ; \xi) /(1-G(x ; \xi))$ of a baseline distribution, where $\xi$ is the parameter vector of this baseline distribution. Then the cdf of the Modified Burr III G Family is defined by

$$
\begin{gathered}
F(x ; \alpha, \beta, \gamma, \xi)=\int_{0}^{H(x ; \xi)}\left[1+\gamma t^{-\beta}\right]^{-\frac{\alpha}{\gamma}} d t \\
F(x ; \alpha, \beta, \gamma, \xi)=\left[1+\gamma(H(x ; \xi))^{-\beta}\right]^{-\frac{\alpha}{\gamma}} x \in D \subseteq \square, \alpha, \beta, \gamma>0
\end{gathered}
$$


The pdf of the Modified Burr III G family reduces to

$$
f(x ; \alpha, \beta, \gamma, \xi)=\alpha \beta(H(x ; \xi))^{-\beta+1}\left[1+\gamma(H(x ; \xi))^{-\beta}\right]^{-\frac{\alpha}{\gamma}-1} \frac{g(x ; \xi)}{(G(x ; \xi))^{2}}, x>0
$$

Here $\mathrm{F}(\mathrm{x} ; \alpha, \beta, \gamma, \xi)$ and $\mathrm{f}(\mathrm{x} ; \alpha, \beta, \gamma, \xi)$ denotes the cumulative distribution function and probability density function respectively of the Modified Burr III G family whereas $\mathrm{G}(\mathrm{x} ; \xi), \mathrm{g}(\mathrm{x} ; \xi)$ and $\mathrm{H}(\mathrm{x} ; \xi)$ are the cdf, pdf and odds ratio of the baseline distribution respectively . The survival function of Modified Burr III G family is defined as

$$
S(x ; \alpha, \beta, \gamma, \xi)=1-\left[1+\gamma(H(x ; \xi))^{-\beta}\right]^{-\frac{\alpha}{\gamma}}
$$

and hazard rate function is

$$
h(x ; \alpha, \beta, \gamma, \xi)=\frac{\alpha \beta(H(x ; \xi))^{-\beta+1}\left[1+\gamma(H(x ; \xi))^{-\beta}\right]^{-\frac{\alpha}{\gamma}-1} \frac{g(x ; \xi)}{(G(x ; \xi))^{2}}}{1-\left[1+\gamma(H(x ; \xi))^{-\beta}\right]^{-\frac{\alpha}{\gamma}}} .
$$

\section{Limiting cases of the family}

For $\gamma \circledR 0$ MBIII G family tends to Generalized Inverse Weibull G family.

For $\beta=1$ and $\gamma \AA 0$ MBIII G family tends to Generalized Inverse Exponential G family.

When $\beta=1, \alpha=1, \gamma{ }^{\circledR} 0$ MBIII G family approaches Inverse exponential G family of distributions. For $\alpha=1, \gamma ® 0$ Inverse Weibull $\mathrm{G}$ family of distributions is a limiting case of MBIII G family.

All of the above mentioned families of distributions are limiting case of Modified Burr III G family. Therefore the proposed family is a generalized family of distributions and all the limiting cases are new families of distributions

\section{Special Models of MBIII G distribution}

In this section we give some examples of Modified Burr III $\mathrm{G}$ family of distributions. These special models generalize several classical models, for example, log-logistic, Lomax, exponential, Uniform among other distributions.

\subsection{Modified Burr III Normal distribution}

First example refers to the Normal distribution. The Modified Burr III Normal (MBIII N) density is obtained from (2.1) by taking $\mathrm{G}(\mathrm{x})$ and $\mathrm{g}(\mathrm{x})$ to be the cdf and pdf of the normal $\mathrm{N}(\mu, \mathrm{s} 2)$ distribution. The MBIII N distribution has cdf

$$
F_{\text {MBIIIN }}(x)=\left[1+\gamma\left(\frac{\Phi\left(\frac{x-\mu}{\sigma}\right)}{1-\Phi\left(\frac{x-\mu}{\sigma}\right)}\right)^{-\beta}\right]^{-\frac{\alpha}{\gamma}},-\infty<x<\infty,
$$


where $-\infty<\mu<\infty, \sigma>0$ and $\mathrm{f}($.$) and \mathrm{F}($.$) are the pdf and cdf of the standard normal distribution$ respectively. Its corresponding pdf is

$$
\begin{aligned}
f_{\text {MBIII N }}(x)=\alpha \beta\left(\frac{\Phi\left(\frac{x-\mu}{\sigma}\right)}{1-\Phi\left(\frac{x-\mu}{\sigma}\right)}\right)^{-\beta+1}\left[1+\gamma\left(\frac{\Phi\left(\frac{x-\mu}{\sigma}\right)}{1-\Phi\left(\frac{x-\mu}{\sigma}\right)}\right)^{-\beta}\right]^{-\frac{\alpha}{\gamma}-1} \frac{\phi\left(\frac{x-\mu}{\sigma}\right)}{\left(\Phi\left(\frac{x-\mu}{\sigma}\right)\right)^{2}}, \\
-\infty<x<\infty .
\end{aligned}
$$

\subsection{Modified Burr III Weibull Distribution}

The Weibull distribution has pdf $g(x)=\lambda \nu x^{\nu-1} \exp \left(-\lambda x^{\nu}\right), x>0$ and cdf $G(x)=1-\exp \left(-\lambda x^{v}\right)$ with odds ratio $H(x)=\exp \left(\lambda x^{v}\right)-1$. Here $\lambda$ is rate and $v$ is scale parameter.

Then MB III Weibull distribution has cdf $F_{\text {MBIII }}(x)=\left[1+\gamma\left(e^{\lambda x^{\nu}}-1\right)^{-\beta}\right]^{-\frac{\alpha}{\gamma}}$ with its pdf as $f_{\text {MBIII W }}(x)=\alpha \beta\left(e^{\lambda x^{\nu}}-1\right)^{-\beta-1}\left[1+\gamma\left(e^{\lambda x^{\nu}}-1\right)^{-\beta}\right]^{-\frac{\alpha}{\gamma}-1} \lambda v x^{\nu-1} e^{\lambda x^{\prime}}, x>0$.

\subsection{Modified Burr III Kumaraswamy Distribution}

Consider the Kumaraswamy distribution with pdf $g(x)=a b x^{a-1}\left(1-x^{a}\right)^{b-1}, 0<x<1$ and cdf as $G(x)=1-\left(1-x^{a}\right)^{b}$ with odds ratio $H(x)=\left(1-x^{a}\right)^{-b}-1$. Where a and $\mathrm{b}$ are its shape parameters.

Then MB III Kumaraswamy distribution has cdf and pdf respectively as $F_{\text {MBII Kим }}(x)=\left[1+\gamma\left\{\left(1-x^{a}\right)^{-b}-1\right\}^{-\beta}\right]^{-\frac{\alpha}{\gamma}}$

$f_{\text {MвІІІ Ким }}(x)=\alpha \beta\left\{\left(1-x^{a}\right)^{-b}-1\right\}^{-\beta-1}\left[1+\gamma\left\{\left(1-x^{a}\right)^{-b}-1\right\}^{-\beta}\right]^{-\frac{\alpha}{\gamma}-1} a b x^{a-1}\left(1-x^{a}\right)^{-b-1}, x>0$.

\subsection{Modified Burr III Burr XII distribution} is

The pdf of Burr XII is $g(x)=c k x^{c-1}\left(1+x^{c}\right)^{-k-1}$, where $\mathrm{c}$ and $\mathrm{k}$ are shape parameters. Its cdf

$$
G(x)=1-\left(1+x^{c}\right)^{-k}
$$

and its odds ratio be 


$$
H(x)=\left(1+x^{c}\right)^{k}-1 .
$$

Then MBIII BXII is defined as

$$
f_{\text {MBII BXII }}(x)=\alpha \beta c k x^{c-1}\left(1+x^{c}\right)^{k-1}\left[\left(1+x^{c}\right)^{k}-1\right]^{-\beta-1}\left[1+\gamma\left\{\left(1+x^{c}\right)^{k}-1\right\}^{-\beta}\right]^{-\frac{\alpha}{\gamma}-1}, x>0 .
$$

With corresponding cdf

$$
F_{\text {MBII BXII }}(x)=\left[1+\gamma\left\{\left(1+x^{c}\right)^{k}-1\right\}^{-\beta}\right]^{-\frac{\alpha}{\gamma}} .
$$

The MBIII BXII distribution includes $\log$-logistic when $\alpha=\beta=\gamma=1$ and $\mathrm{k}=1$ with pdf

$$
f(x)=c x^{c-1}\left(1+x^{c}\right)^{-2} .
$$

For $\alpha=\beta=\gamma=1$ and $c=1$ we obtain Lomax distribution with pdf

$$
f(x)=k(1+x)^{k-1} \text {. }
$$

And when all the Modified Burr III parameters are one we get Burr XII distribution with pdf

$$
f(x)=c k x^{c-1}\left(1+x^{c}\right)^{-k-1} .
$$

To study the shape of the Modified Burr III G family of distributions we take some selected values of the parameters of the above mentioned special models to plot their densities and hazard rate function.

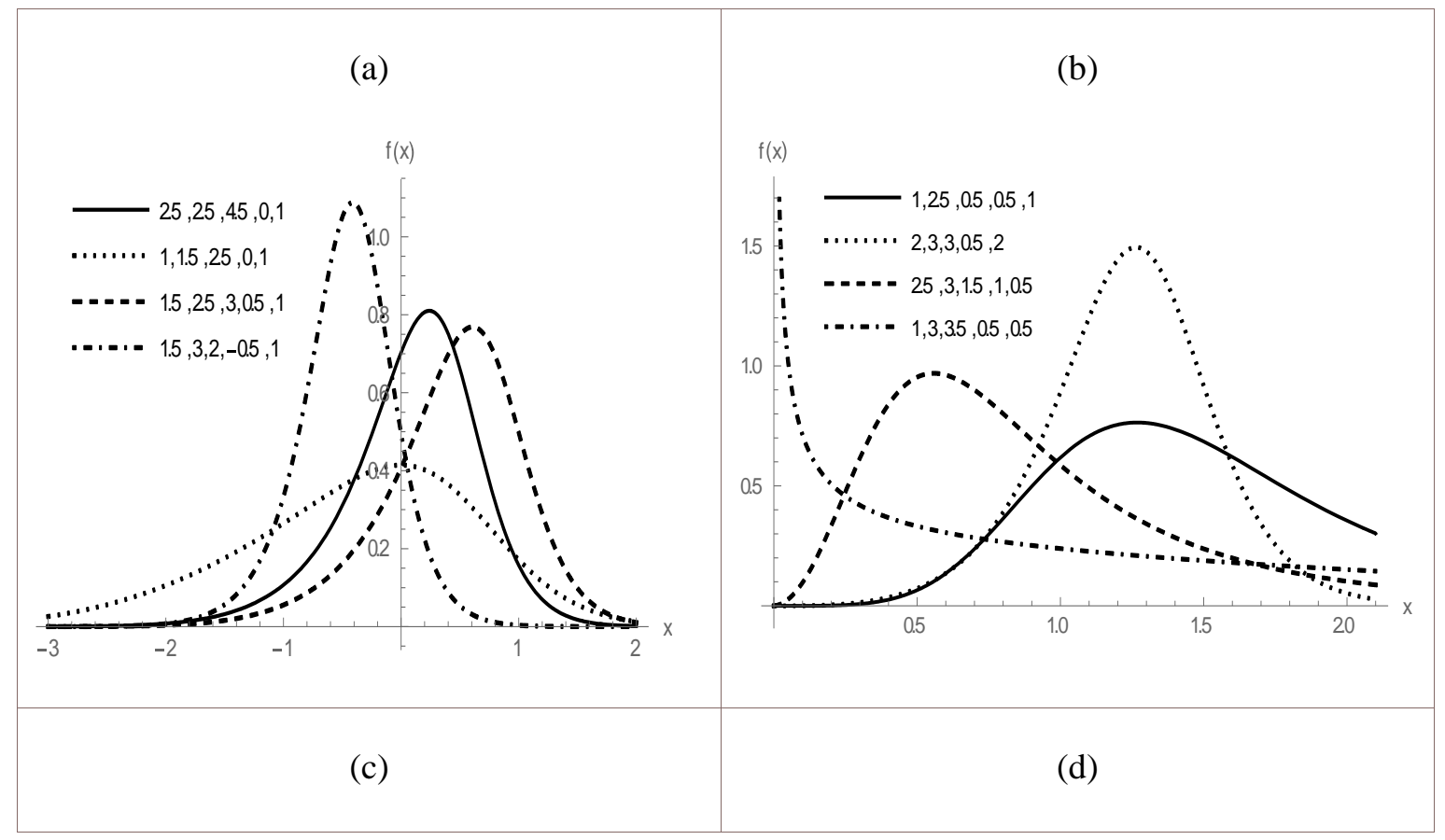




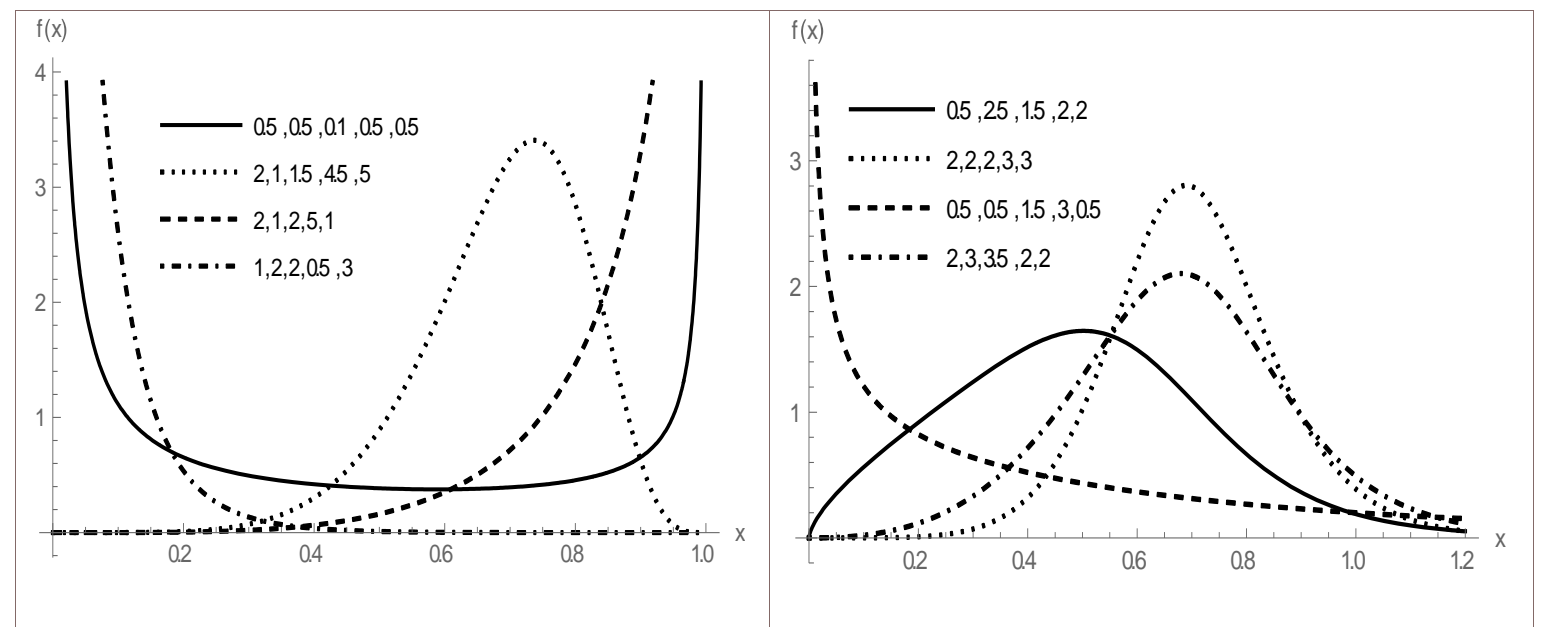

Figure 1: Plots of the densities of (a) MBIII Normal (b) MBIII Weibull (c) MBIII Kumaraswamy and (d) MBIII Burr XII distributions for various values of the parameters.

Figure 1 indicates that the proposed Modified Burr III G family generates distributions with various shapes such as bell-shaped, left-skewed, right-skewed, bathtub, J and reversed-J. It is a clear indication that the MBIII G family is very flexible and can be used appropriately to fit different datasets having various shapes.

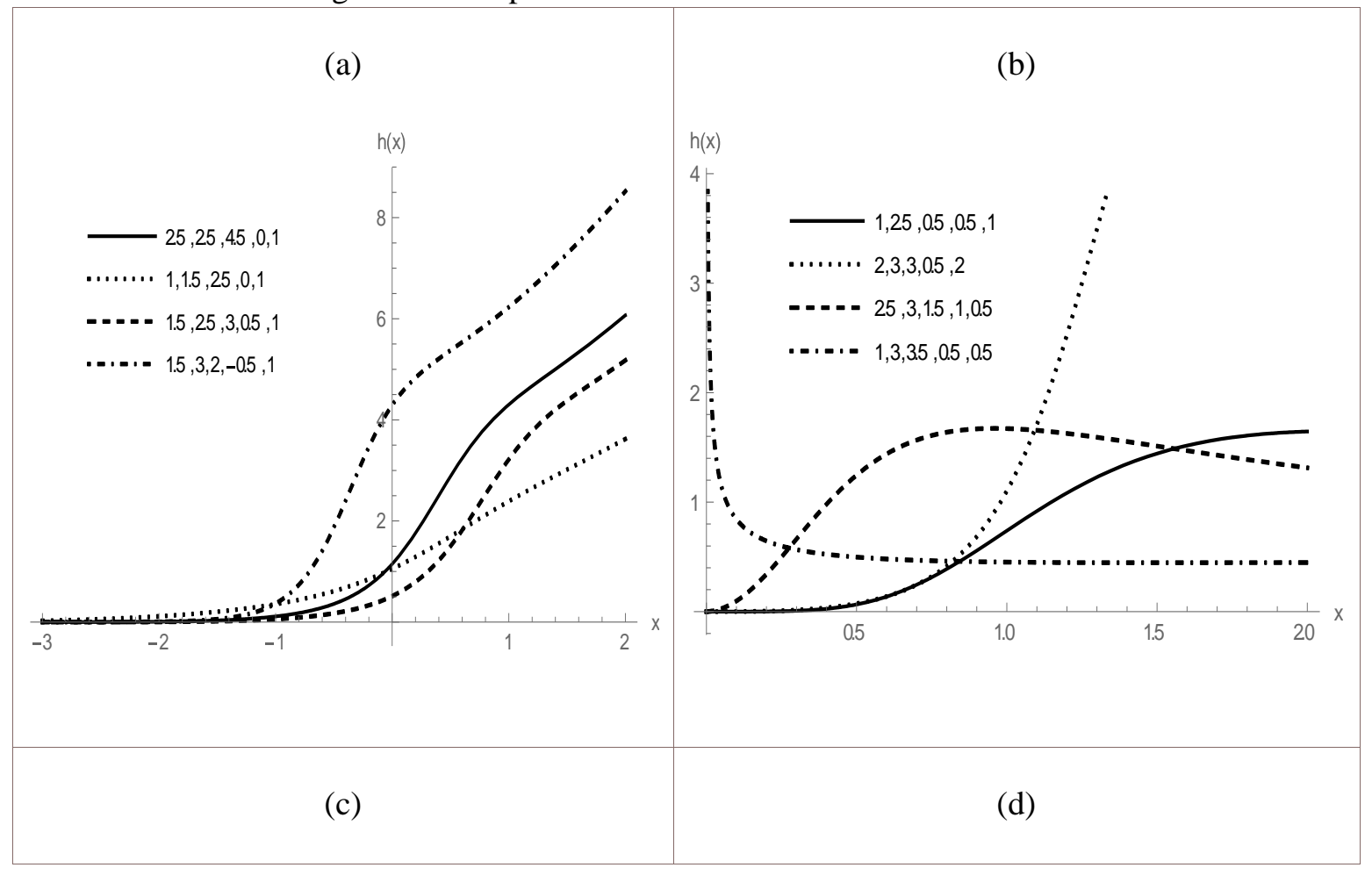




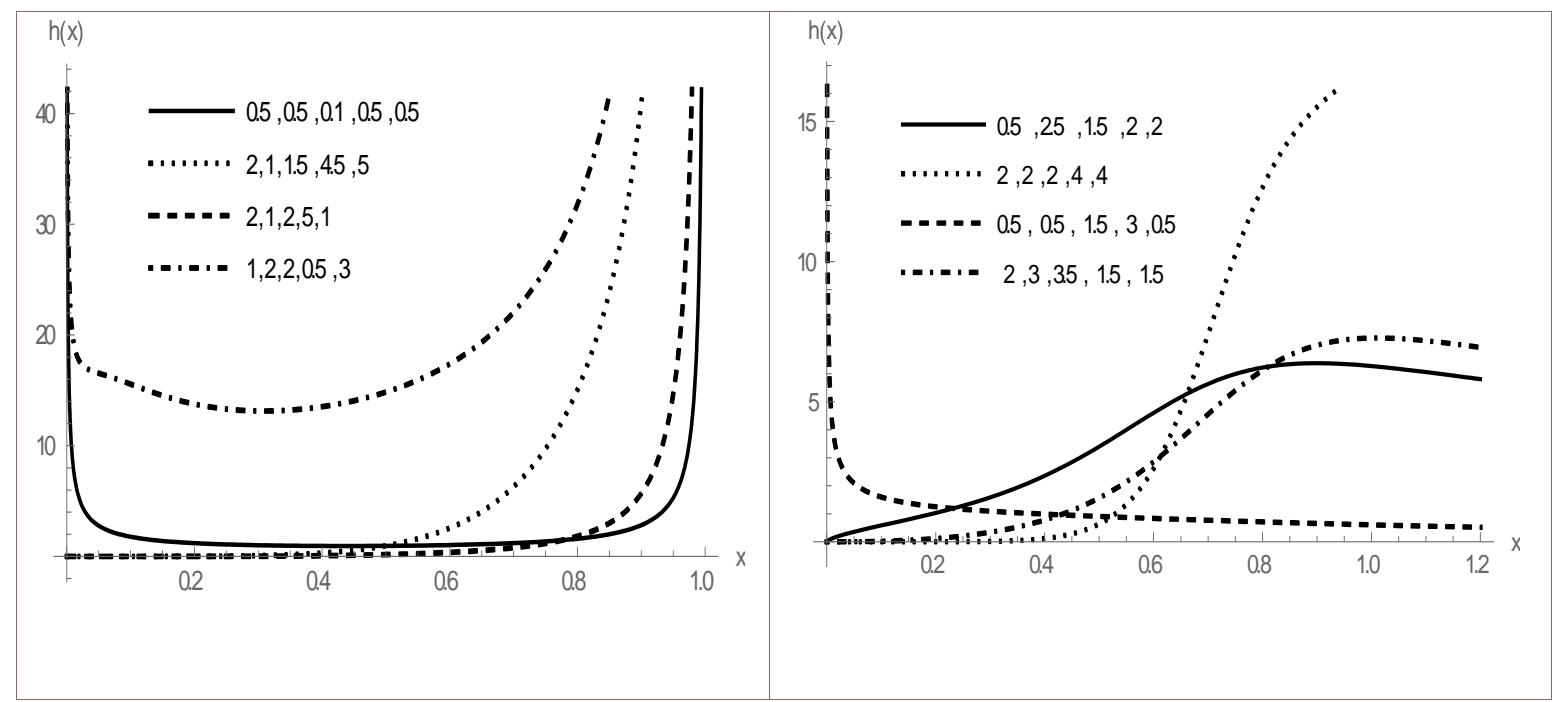

Figure 2: Plots of the hazard rate of (a) MBIII Normal (b) MBIII Weibull (c) MBIII Kumaraswamy and (d) MBIII Burr XII distributions for various values of the parameters.

Figure 2 shows that the hazard rate of Modified Burr III $G$ family of distributions can be monotonic or non-monotonic. The shapes of hazard rate can be increasing, decreasing, bathtub, upside-down bathtub, $\mathrm{J}$ and reversed-J. This is indicative of its usefulness in survival analysis, biomedical, engineering and social sciences.

\section{Mathematical Properties}

We now derive some of the general mathematical properties of Modified Burr III G distribution.

\subsection{Expansion for the Modified Burr III G family of distributions}

Expansion for the pdf of MBIII $G$ family can be derived by using the binomial series expansion in (2.2). Let $\mathrm{f}(\mathrm{x} ; \alpha, \beta, \gamma ; \xi)=\mathrm{f}(\mathrm{x})$

$$
f(x)=\alpha \beta(H(x))^{-\beta+1}\left[1+\gamma(H(x))^{-\beta}\right]^{-\frac{\alpha}{\gamma}-1} \frac{g(x)}{(G(x))^{2}}
$$

Using binomial expansion for the second term in the above equation we have

$$
\left[1+\gamma(H(x))^{-\beta}\right]^{-\frac{\alpha}{\gamma}-1}=\sum_{k=0}^{\infty}(-1)^{k} \gamma^{k}\left(\begin{array}{c}
\frac{\alpha}{\gamma}+k \\
k
\end{array}\right)(H(x))^{-\beta k}
$$

As $H(x)=G(x) /(1-G(x))$ equation (4.1.1) becomes 


$$
f(x)=\alpha \beta \sum_{k=0}^{\infty}(-1)^{k} \gamma^{k}\left(\begin{array}{c}
\frac{\alpha}{\gamma}+k \\
k
\end{array}\right) \frac{(G(x))^{-\beta(k+1)-1}}{(1-G(x))^{-\beta(k+1)+1}} g(x)
$$

Using binomial series again in (4.1.3)

$$
(1-G(x))^{\beta(k+1)-1}=\sum_{j=0}^{\infty}(-1)^{j}\left(\begin{array}{c}
\beta(k+1)-1 \\
j
\end{array}\right)(G(x))^{j}
$$

and substituting in (4.1.3) we get

$$
f(x)=\alpha \beta \sum_{j, k=0}^{\infty}(-1)^{k+j} \gamma^{k}\left(\begin{array}{c}
\frac{\alpha}{\gamma}+k \\
k
\end{array}\right)\left(\begin{array}{c}
\beta(k+1)-1 \\
j
\end{array}\right)(G(x))^{j-\beta(k+1)-1} g(x)
$$

The above equation can be rewritten as

$$
f(x)=\sum_{k=0}^{\infty} w_{j, k} h_{j-\beta(k+1)-1}(x)
$$

where

$$
w_{j, k}=\alpha \beta \frac{(-1)^{k+j} \gamma^{k} \Gamma\left(\frac{\alpha}{\gamma}+k+1\right) \Gamma(\beta(k+1))}{k ! j ! \Gamma\left(\frac{\alpha}{\gamma}+1\right) \Gamma(\beta(k+1)-j)[j-\beta(k+1)-1]}
$$

and

$$
h_{a}(x)=a g(x)(G(x))^{a-1} .
$$

In other words we can write the MBIII G family density as a linear combination of exponentiated-G density (exp-G) function with weights $w_{j, k}$. Therefore some mathematical properties of the proposed family can be derived from (4.1.5) similar to those of exp-G properties such as the ordinary and incomplete moments and moment generating function. For mathematical properties of the exp-G distributions the reader is referred to Gupta and Kundu (2001), Gupta et al. (1998) and Nadarajah and Kotz(2006) among others.

\subsection{Moments}

The sth moment of Modified Burr III G family of distributions can be obtained from (4.1.5) as it can be expressed as an infinite linear combination of the exponentiated-G density functions

$$
f(x)=\sum_{k=0}^{\infty} w_{j, k} h_{j-\beta(k+1)-1}(x)
$$

as

$$
E\left(X^{s}\right)=\sum_{j, k=0}^{\infty} w_{j, k} E\left(Z_{j, k}^{s}\right)
$$


where $Z_{j, k}$ denotes the exponentiated-G distribution with power parameter $j-\beta(k+1)-1$. The inner quantities in (4.1.5) are absolutely integrable.

The incomplete moments and moment generating function can be evaluated as

$$
I_{X}(y)=\int_{-\infty}^{y} x^{s} f(x) d x=\sum_{j, k=0}^{\infty} w_{j, k} I_{j, k}(y)
$$

where

$$
I_{j, k}(y)=\int_{-\infty}^{y} x^{s} h_{j-\beta(k+1)-1}(x ; \xi) d x
$$

MGF is

$$
M_{X}(t)=\sum_{j, k=0}^{\infty} w_{j, k} E\left(e^{t z_{j, k}}\right)
$$

\subsection{Quantile function and Random Number Generation}

The Modified Burr III G family of distributions can easily be simulated from (2.6) as follows. If ' $\mathrm{q}$ ' has a uniform distribution $\mathrm{U}(0,1)$, the solution

$$
X=G^{-1}(S /(S+1)) \text { has the MB III G }
$$

distribution where $S=\left[\gamma /\left(q^{-\frac{\gamma}{\alpha}}-1\right)\right]^{1 / \beta}$. For q=0.5 we get median of MB III G distribution and
random number generator if $\mathrm{q}$ is taken as uniform.

\section{Maximum Likelihood Estimation}

Now we find the maximum likelihood estimates (MLEs) of the unknown parameters of the Modified Burr III $\mathrm{G}$ family of distributions. Let $\mathrm{x} 1, \mathrm{x} 2, \ldots, \mathrm{xn}$ be a sample of size $\mathrm{n}$ from MBIII $\mathrm{G}$ family given by (2.2) with parameters $\alpha, \beta, \gamma$ and $\xi$. Let $\mathrm{Q}=(\alpha, \beta, \gamma, \xi) \mathrm{T}$

be the $\mathrm{p}$ x 1 parameter vector. Then log-likelihood function can be expressed as

$$
\begin{aligned}
l(\Theta)= & n \log \alpha+n \log \beta-(\beta+1) \sum_{i=1}^{n} \log \left[G\left(x_{i} ; \xi\right)\right]+(\beta-1) \sum_{i=1}^{n} \log \left[1-G\left(x_{i} ; \xi\right)\right] \\
& -\left(\frac{\alpha}{\gamma}+1\right) \sum_{i=1}^{n} \log \left[1+\gamma\left(H\left(x_{i} ; \xi\right)\right)^{-\beta}\right]+\sum_{i=1}^{n} \log \left(g\left(x_{i} ; \xi\right)\right)
\end{aligned}
$$

Where $\mathrm{H}(\mathrm{x} ; \xi)=\mathrm{G}(\mathrm{x} ; \xi) /(1-\mathrm{G}(\mathrm{x} ; \xi))$. The components of the score function $\mathrm{U}(\mathrm{Q})=(\mathrm{U} \alpha, \mathrm{U} \beta, \mathrm{U} \gamma, \mathrm{U} \xi \mathrm{k})$ are given by

$$
\begin{gathered}
U_{\alpha}=\frac{n}{\alpha}-\frac{1}{\gamma} \sum_{i=1}^{n} \log \left[1+\gamma\left(H\left(x_{i} ; \xi\right)\right)^{-\beta}\right] \\
U_{\beta}=\frac{n}{\beta}-\sum_{i=1}^{n} \log \left[G\left(x_{i} ; \xi\right)\right]+\sum_{i=1}^{n} \log \left[1-G\left(x_{i} ; \xi\right)\right]+\left(\frac{\alpha}{\gamma}+1\right) \sum_{i=1}^{n} \frac{\gamma \log H\left(x_{i} ; \xi\right)\left(H\left(x_{i} ; \xi\right)\right)^{-\beta}}{\left[1+\gamma\left(H\left(x_{i} ; \xi\right)\right)^{-\beta}\right]}
\end{gathered}
$$




$$
U_{\gamma}=\frac{\alpha}{\gamma^{2}} \sum_{i=1}^{n} \log \left[1+\gamma\left(H\left(x_{i} ; \xi\right)\right)^{-\beta}\right]-\left(\frac{\alpha}{\gamma}+1\right) \sum_{i=1}^{n} \frac{\left(H\left(x_{i} ; \xi\right)\right)^{-\beta}}{\left[1+\gamma\left(H\left(x_{i} ; \xi\right)\right)^{-\beta}\right]}
$$

and

$$
\begin{aligned}
U_{\xi_{k}}= & -(\beta+1) \sum_{i=1}^{n}\left[\frac{\partial G\left(x_{i} ; \xi\right) / \partial \xi_{k}}{G\left(x_{i} ; \xi\right)}\right]+(\beta-1) \sum_{i=1}^{n}\left[\frac{\partial \bar{G}\left(x_{i} ; \xi\right) / \partial \xi_{k}}{\bar{G}\left(x_{i} ; \xi\right)}\right] \\
& +\left(\frac{\alpha}{\gamma}+1\right) \sum_{i=1}^{n}\left[\frac{\gamma \beta\left(H\left(x_{i} ; \xi\right)\right)^{-\beta-1} \partial H\left(x_{i} ; \xi\right) / \partial \xi_{k}}{\left[1+\gamma\left(H\left(x_{i} ; \xi\right)\right)^{-\beta}\right]}\right]+\sum_{i=1}^{n} \frac{\partial g\left(x_{i} ; \xi\right) / \partial \xi_{k}}{g\left(x_{i} ; \xi\right)}
\end{aligned}
$$

Setting these score equations equal to zero and solving them simultaneously yields the MLE $\hat{\Theta}=(\alpha, \beta, \gamma, \xi)^{T}$ of $\Theta=(\alpha, \beta, \gamma, \xi)^{T}$. These equations cannot be solved analytically and recourse to iterative procedure such as Newton-Raphson algorithm is suggested. For interval estimation of the model parameters, we need the observed information matrix

$$
J(\Theta)=\left(\begin{array}{ccccc}
U_{\alpha \alpha} & U_{\alpha \beta} & U_{\alpha \gamma} & \mid & U_{\alpha \xi} \\
U_{\beta \alpha} & U_{\beta \beta} & U_{\beta \gamma} & \mid & U_{\beta \xi} \\
U_{\gamma \alpha} & U_{\gamma \beta} & U_{\gamma \gamma} & \mid & U_{\gamma \xi} \\
-- & -- & -- & -- & -- \\
U_{\xi \alpha} & U_{\xi \beta} & U_{\xi \gamma} & \mid & U_{\xi \xi}
\end{array}\right)
$$

Whose elements are

$$
\begin{aligned}
& U_{\alpha \alpha}=-\frac{n}{\alpha^{2}} \\
& U_{\alpha \beta}=\sum_{i=1}^{n} \frac{\left(H\left(x_{i} ; \xi\right)\right)^{-\beta} \log H\left(x_{i} ; \xi\right)}{\left[1+\gamma\left(H\left(x_{i} ; \xi\right)\right)^{-\beta}\right]} \\
& U_{\alpha \gamma}=\frac{1}{\gamma^{2}} \sum_{i=1}^{n} \log \left[1+\left(H\left(x_{i} ; \xi\right)\right)^{-\beta}\right]-\frac{1}{\gamma} \sum_{i=1}^{n} \frac{\left(H\left(x_{i} ; \xi\right)\right)^{-\beta}}{\left[1+\gamma\left(H\left(x_{i} ; \xi\right)\right)^{-\beta}\right]}
\end{aligned}
$$




$$
\begin{aligned}
& U_{\alpha \xi}=\sum_{i=1}^{n} \frac{\beta\left(H\left(x_{i} ; \xi\right)\right)^{-\beta-1}}{\left[1+\gamma\left(H\left(x_{i} ; \xi\right)\right)^{-\beta}\right]} H_{k}^{\prime}\left(x_{i} ; \xi\right) \\
& U_{\beta \beta}=-\frac{n}{\beta^{2}}-\left(\frac{\alpha}{\gamma}+1\right) \sum_{i=1}^{n} \frac{\gamma\left(\log H\left(x_{i} ; \xi\right)\right)^{2}\left(H\left(x_{i} ; \xi\right)\right)^{-\beta}}{\left[1+\gamma\left(H\left(x_{i} ; \xi\right)\right)^{-\beta}\right]^{2}} \\
& U_{\beta \gamma}=-\sum_{i=1}^{n} \frac{\log H\left(x_{i} ; \xi\right)\left(H\left(x_{i} ; \xi\right)\right)^{-\beta}}{\left[1+\gamma\left(H\left(x_{i} ; \xi\right)\right)^{-\beta}\right]} \\
& U_{\beta \xi_{k}}=-\sum_{i=1}^{n} \frac{G_{k}^{\prime}\left(x_{i} ; \xi\right)}{G\left(x_{i} ; \xi\right)}-\sum_{i=1}^{n} \frac{G_{k}^{\prime}\left(x_{i} ; \xi\right)}{1-G\left(x_{i} ; \xi\right)}+ \\
& \left(\frac{\alpha}{\gamma}+1\right) \sum_{i=1}^{n} \frac{\gamma\left(H\left(x_{i} ; \xi\right)\right)^{-\beta-1}\left[1+\gamma\left(H\left(x_{i} ; \xi\right)\right)^{-\beta}-\beta \log H\left(x_{i} ; \xi\right)\right] \partial H_{k}^{\prime}\left(x_{i} ; \xi\right)}{\left[1+\gamma\left(H\left(x_{i} ; \xi\right)\right)^{-\beta}\right]^{2}} \\
& U_{\gamma}=-\frac{2 \alpha}{\gamma^{3}} \sum_{i=1}^{n} \log \left[1+\left(H\left(x_{i} ; \xi\right)\right)^{-\beta}\right]-\frac{2 \alpha}{\gamma^{2}} \sum_{i=1}^{n} \frac{\left(H\left(x_{i} ; \xi\right)\right)^{-\beta}}{\left[1+\gamma\left(H\left(x_{i} ; \xi\right)\right)^{-\beta}\right]} \\
& +\left(\frac{\alpha}{\gamma}+1\right) \sum_{i=1}^{n} \frac{\left(H\left(x_{i} ; \xi\right)\right)^{-2 \beta}}{\left[1+\gamma\left(H\left(x_{i} ; \xi\right)\right)^{-\beta}\right]^{2}} \\
& U_{\gamma \xi_{k}}=\sum_{i=1}^{n} \frac{\beta\left(H\left(x_{i} ; \xi\right)\right)^{-\beta-1}\left[1+\alpha\left(H\left(x_{i} ; \xi\right)\right)^{-\beta}\right] H_{k}^{\prime}\left(x_{i} ; \xi\right)}{\left[1+\gamma\left(H\left(x_{i} ; \xi\right)\right)^{-\beta}\right]^{2}}
\end{aligned}
$$

and 


$$
\begin{aligned}
& U_{\xi_{k} \xi_{l}}=-(\beta+1) \sum_{i=1}^{n} \frac{G_{k l}^{\prime \prime}\left(x_{i} ; \xi\right)}{G\left(x_{i} ; \xi\right)}+(\beta+1) \sum_{i=1}^{n} \frac{G_{k}^{\prime}\left(x_{i} ; \xi\right) G_{l}^{\prime}\left(x_{i} ; \xi\right)}{\left(G\left(x_{i} ; \xi\right)\right)^{2}}+(\beta+1) \sum_{i=1}^{n} \frac{\bar{G}_{k l}^{\prime \prime}\left(x_{i} ; \xi\right)}{\bar{G}\left(x_{i} ; \xi\right)} \\
& -(\beta+1) \sum_{i=1}^{n} \frac{\bar{G}_{k}^{\prime}\left(x_{i} ; \xi\right) \bar{G}_{l}^{\prime}\left(x_{i} ; \xi\right)}{\left(G\left(x_{i} ; \xi\right)\right)^{2}}+\sum_{i=1}^{n} \frac{g^{\prime \prime}{ }_{k l}\left(x_{i} ; \xi\right)}{g\left(x_{i} ; \xi\right)}-\sum_{i=1}^{n} \frac{g_{k}^{\prime}\left(x_{i} ; \xi\right) g_{l}^{\prime}\left(x_{i} ; \xi\right)}{\left(g\left(x_{i} ; \xi\right)\right)^{2}} \\
& +\left(\frac{\alpha}{\gamma}+1\right) \sum_{i=1}^{n} \gamma \beta\left[\frac{\left(H\left(x_{i} ; \xi\right)\right)^{-\beta-1} H_{k l}^{\prime \prime}\left(x_{i} ; \xi\right)}{\left[1+\gamma\left(H\left(x_{i} ; \xi\right)\right)^{-\beta}\right]}\right]+\left(\frac{\alpha}{\gamma}+1\right) \sum_{i=1}^{n} \gamma \beta\left[\frac{(\beta+1)\left(H\left(x_{i} ; \xi\right)\right)^{-\beta-2} H_{k}^{\prime}\left(x_{i} ; \xi\right) H_{l}^{\prime}\left(x_{i} ; \xi\right)}{\left[1+\gamma\left(H\left(x_{i} ; \xi\right)\right)^{-\beta}\right]^{2}}\right] \\
& -\left(\frac{\alpha}{\gamma}+1\right) \sum_{i=1}^{n} \gamma \beta\left[\frac{\gamma\left(H\left(x_{i} ; \xi\right)\right)^{-2 \beta-2} H_{k}^{\prime}\left(x_{i} ; \xi\right) H_{l}^{\prime}\left(x_{i} ; \xi\right)}{\left[1+\gamma\left(H\left(x_{i} ; \xi\right)\right)^{-\beta}\right]^{2}}\right] .
\end{aligned}
$$

Where $G_{k}^{\prime}\left(x_{i} ; \xi\right)$ and $G_{k l}^{\prime \prime}\left(x_{i} ; \xi\right)$ denotes first and second derivative of xi with respect to $\xi$.

\section{Applications}

In this section, we provide two applications to real data to illustrate the importance of the Modified Burr III G family by means of Modified Burr III Weibull (MBIII Wei) and Modified Burr III Burr XII (MBIII BXII) models presented in Section 3.

\section{Application 1}

The first data consists of 63 observations of the strengths of $1.5 \mathrm{~cm}$ glass fibers. It was originally obtained be workers at the UK National Physical Laboratory. These data have also been analyzed by Smith and Naylor (1987). The units of measurement are not given in the paper. The data are:

$0.55,0.74,0.77,0.81,0.84,0.93,1.04,1.11,1.13,1.24,1.25,1.27,1.28,1.29,1.30,1.36,1.39$, $1.42,1.48,1.48,1.49,1.49,1.50,1.50,1.51,1.52,1.53,1.54,1.55,1.55,1.58,1.59,1.60,1.61$, $1.61,1.61,1.61,1.62,1.62,1.63,1.64,1.66,1.66,1.66,1.67,1.68,1.68,1.69,1.70,1.70,1.73$, $1.76,1.76,1.77,1.78,1.81,1.82,1.84,1.84,1.89,2.00,2.01,2.24$.

For this data we compare Modified Burr III Weibull distribution with Modified Burr III and Weibull model.

Table 1: Summary statistics of strengths of $1.5 \mathrm{~cm}$ glass fibers data

\begin{tabular}{|l|l|l|l|l|l|l|}
\hline Data & Mean & Median & S.D & Variance & Skewness & Kurtosis \\
\hline Strength of glass fibers & 1.359 & 1.59 & 1.19501 & 1.42805 & -0.8999 & 3.9238 \\
\hline
\end{tabular}


The required numerical calculations of MLEs, negative log-likelihood (W), Akaike Information Criterion (AIC), Cumulative Akaike Information Criterion (CAIC) and Bayesian Information Criterion (BIC) values are carried out using SAS through PROC NLMIXED command. Using several different initial values of the parameters we find the best fit for each model. The initial guess that gives the minimum value of the likelihood criterion is considered to be the best fit of parameter estimates. Following results were obtained.

Table 2: MLEs of the parameters, their standard errors and various information criteria for the strengths of $1.5 \mathrm{~cm}$ glass fibers dataset

\begin{tabular}{|c|c|c|c|c|c|c|c|c|}
\hline Model & estimates & S.E. & $\begin{array}{l}\text { Lower } \\
\text { bound }\end{array}$ & $\begin{array}{l}\text { Upper } \\
\text { bound }\end{array}$ & W & AIC & CAIC & BIC \\
\hline \multirow{5}{*}{$\begin{array}{c}\text { MBIII } \\
\text { Weibull }\end{array}$} & $\hat{\alpha}=257.01$ & 1360.65 & -2462.04 & 2976.05 & \multirow{5}{*}{22.6} & \multirow{5}{*}{32.6} & \multirow{5}{*}{33.7} & \multirow{5}{*}{43.3} \\
\hline & $\hat{\beta}=3.238$ & 5.59 & -7.94 & 14.41 & & & & \\
\hline & $\hat{\gamma}=660$ & 3476.19 & -6286.62 & 7606.61 & & & & \\
\hline & $\hat{\lambda}=0.646$ & 1.05 & -1.45 & 2.74 & & & & \\
\hline & $\hat{v}=2.19$ & 1.42 & -0.64 & 5.03 & & & & \\
\hline \multirow{3}{*}{ MBIII } & $\hat{\alpha}=14400$ & 25696 & -36948 & 65749 & \multirow{3}{*}{24.0} & \multirow{3}{*}{30.0} & \multirow{3}{*}{30.4} & \multirow{3}{*}{36.4} \\
\hline & $\hat{\beta}=19.046$ & 3.11 & 12.82 & 25.27 & & & & \\
\hline & $\hat{\gamma}=59000$ & 116854 & -174514 & 292514 & & & & \\
\hline \multirow{2}{*}{ Weibull } & $\hat{\lambda}=0.059$ & 0.02 & 0.02 & 0.10 & \multirow{2}{*}{30.4} & \multirow{2}{*}{34.4} & \multirow{2}{*}{34.6} & \multirow{2}{*}{38.7} \\
\hline & $\hat{v}=5.78$ & 0.58 & 4.63 & 6.93 & & & & \\
\hline
\end{tabular}

Negative log-likelihood criterion is smaller for MBIII Wei than MBIII and Weibull therefore, it provides better fit than the rest. The CDF plot of these models is given below. 


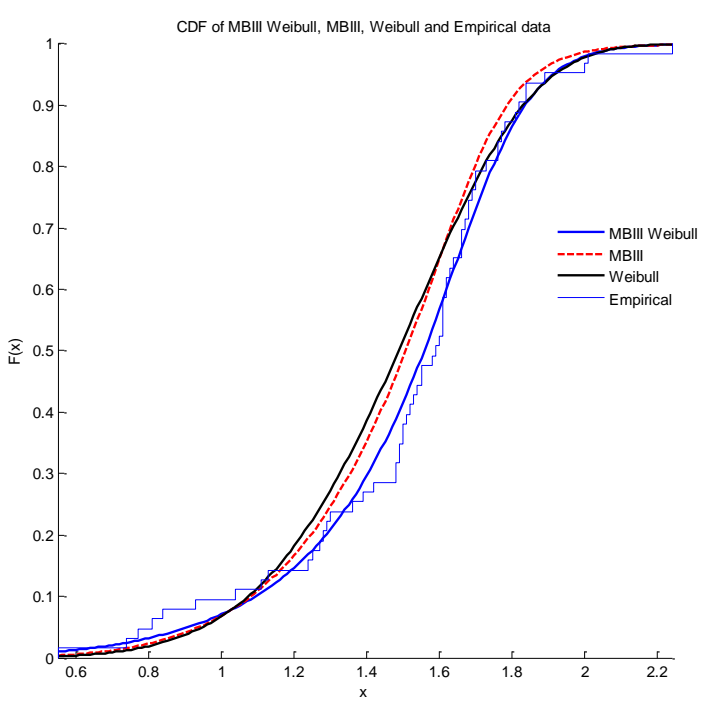

Figure 3: Cdf of MBIII W , MBIII, Weibull and empirical data fitted on glass fiber data

The above plot indicates that Modified Burr III Weibull model gives a close fit of the data than the other models.

\section{Application 2}

In the second application we compare Modified Burr III Burr XII (MBIII BXII) with Modified Burr III (MBIII) and Burr XII (BXII) distribution. We use fracture toughness data (in the units of MPa m1/2) of Alumina (Al2O3) that Nadarajah and Kotz (2007) fit to Burr III (B III) and Burr XII (BXII) distributions. Ali et al. (2014) also applied this data. The data are also available online at http://www.ceramics.nist.gov/srd/summary/ftmain.htm.

Table 3: Summary statistics of Fracture toughness data of Alumina (Al2O3)

\begin{tabular}{|l|l|l|l|l|l|l|}
\hline Data & Mean & Median & S.D & Variance & Skewness & Kurtosis \\
\hline $\begin{array}{l}\text { Fracture Toughness } \\
\text { (in the unit MPa } \mathrm{m}^{1 / 2} \text { ) }\end{array}$ & 4.33 & 4.38 & 1.013 & 1.026 & -0.42 & 3.093 \\
\hline
\end{tabular}

The different information criteria computed are given as.

Table 4: MLEs of the parameters, their standard errors and various information criteria for Fracture toughness data of Alumina (Al2O3) 


\begin{tabular}{|c|c|c|c|c|c|c|c|c|}
\hline Model & estimates & S.E. & $\begin{array}{l}\text { Lower } \\
\text { bound }\end{array}$ & $\begin{array}{l}\text { Upper } \\
\text { bound }\end{array}$ & $\mathrm{W}$ & AIC & CAIC & BIC \\
\hline \multirow{5}{*}{$\begin{array}{c}\text { MBIII } \\
\text { BXII }\end{array}$} & $\hat{\alpha}=141$ & 50.87 & 40.26 & 241.74 & \multirow{5}{*}{335.7} & \multirow{5}{*}{345.7} & \multirow{5}{*}{346.2} & \multirow{5}{*}{359.6} \\
\hline & $\hat{\beta}_{=210}$ & 2024.88 & -3799 & 4219.46 & & & & \\
\hline & $\hat{\gamma}=500$ & 128.39 & 245.78 & 754.22 & & & & \\
\hline & $\hat{c}=0.07$ & 0.69 & -1.30 & 1.44 & & & & \\
\hline & $\hat{k}=0.9$ & 0.56 & -0.17 & 2.06 & & & & \\
\hline \multirow{3}{*}{ MBIII } & $\hat{\alpha}=1111.23$ & 460.94 & 198.52 & 2023.93 & \multirow{3}{*}{373.4} & \multirow{3}{*}{379.4} & \multirow{3}{*}{379.6} & \multirow{3}{*}{387.7} \\
\hline & $\hat{\beta}_{=4.9484}$ & 0.28 & 4.39 & 5.50 & & & & \\
\hline & $\hat{\gamma}=770.7$ & 398.83 & -19.02 & 1560.41 & & & & \\
\hline \multirow{2}{*}{ BXII } & $\hat{c}=22.8$ & 1997.73 & -3933.9 & 3977.5 & \multirow{2}{*}{664.2} & \multirow{2}{*}{668.2} & \multirow{2}{*}{668.3} & \multirow{2}{*}{673.8} \\
\hline & $\hat{k}=0.03$ & 2.04 & -5.78 & 5.84 & & & & \\
\hline
\end{tabular}

Clearly MB III BXII distribution provides best fit among MB III and B XII distributions, because statistic value in all criteria are smaller for MB III BXII distribution. To illustrate the fit of these models we plot the cdfs of these distributions and the empirical data. 


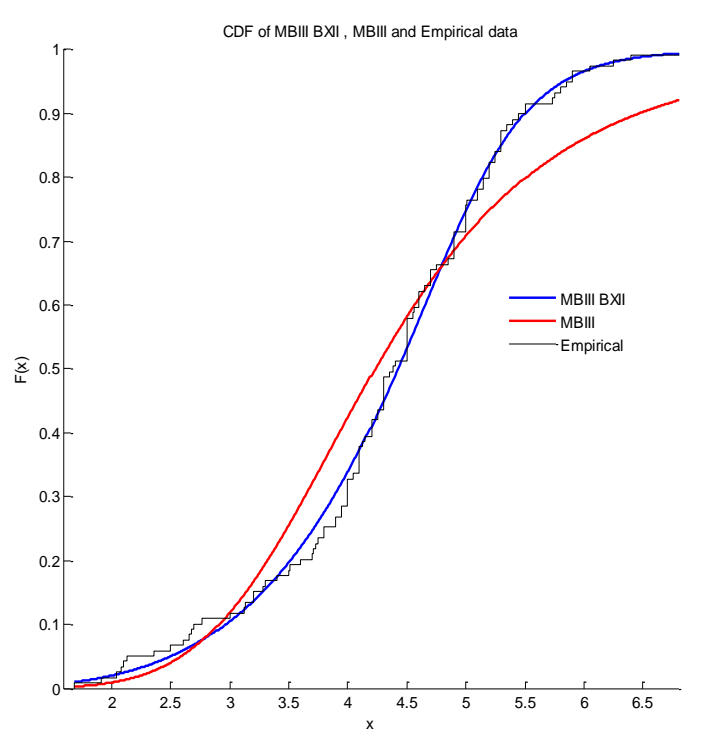

Figure 4: Cdf of MBIII BXII , MBIII, Burr XII distributions and empirical data fitted on fracture toughness data of Alumina (Al2O3)

The fit of the plot shows that MBIII BXII distribution outperforms other models to the empirical data.

Hence we can say that the proposed distribution provides us best fit and is more flexible and has wider application in life time data sets.

\section{Concluding Remarks}

In this paper, we propose a new Modified Burr III G family of distributions. Modified Burr III is the generator wherein the random variable is replaced by the odd ratio of the baseline distribution. We study some of its special distributions. It is observed that the density function can be left-skewed, right-skewed, bell shaped, bathtub, J and reversed-J shaped. We derive some of the general properties of the family and maximum likelihood method is used to estimate its parameters. We fit two models of the new family to demonstrate usefulness and flexibility of the proposed family. We hope that the new family and its models will be useful for practitioners in various fields of applied sciences.

\section{Reference}

[1] Afify, A. Z. , Cordeiro, G. M. , Yousof, H. M. , Nofal, Z. M. and Alzaatreh, A. (2016). The Kumaraswamy Transmuted-G Family of Distributions: Properties and applications. Journal of Data Sciences. 14(2), 245-270. 
[2] Ali, A. , Hasnain, S. A. and Ahmed, M. (2015). Modified Burr III distribution: Properties and applications. Pakistan Journal of Statistics, Vol. 31(6), 697-708.

[3] Alizadeh, M., Emadi, M., Doostparast, M., Cordeiro, G.M., Ortega, E.M.M. and Pescim, R.R.(2015). A new family of distributions: the Kumaraswamy odd log-logistic, properties and applications. Hacettepa Journal of Mathematics and Statistics (to appear).

[4] Alzaatreh, A. , Famoye, F. and Lee, C. (2012). Gamma-Pareto distribution and its applications. Journal of Modern Applied Statistical Methods, 11(1) , 78-94. http://digitalcommons.wayne.edu/jmasm/vol11/iss1/7

[5] Alzaatreh, A. , Lee, C. and Famoye, F. (2013a). A new method for generating families of continuous distributions. Metron . 71(1), 63-79. http:// dx.doi.org/10.1007/s40300-0130007-y

[6] Alzaatreh, A. Famoye, F. and Lee, C. (2013b). Weibull-Pareto distribution and its applications. Communication in Statistics-Theory and Methods, 42(9), 1673-1691. http:// dx.doi.org/10.1080/03610926.2011.599002

[7] Alzaatreh, A. Famoye, F. and Lee, C. (2014). The gamma-normal distribution: Properties and applications. Computational Statistics and Data Analysis, 69(1), 67-80. http:// dx.doi.org/10.1016/j.csda.2013.07.035

[8] Bourguignon, M., Silva, R.B. and Cordeiro, G.M.(2014). The Weibull-G Family of Probability Distributions. Journal of Data Science, 12(1), 53-68.

[9] Burr, I. W. (1942) Cumulative frequency functions. Annals of Mathematical Statistics, 13, 215-232.

[10]Cordeiro, G.M., de Castro, M. (2011).A new family of generalized distributions. Journal of Statistical Computation and Simulation. 81, 883-898.

[11]Cordeiro, G.M., Hashimoto, E.M. and Ortega, E.M.M. (2012). The McDonald Weibull model. Statistics, iFirst, 1-23.

[12]Eugene, N., Lee, C. and Famoya, F. (2002). Beta-Normal distribution and its application. Communication in Statistics-Theory and Methods,31,Issue 4 497-512.

[13] Gove, J. H., Ducey, M. J., Leak, W. B. and Zhang, L. (2008). Rotated sigmoid structure in managed uneven-aged northern hardwood stands: a look at the Burr Type III distribution. International Journal of Forestry Resources., 81(2), 161-175.

[14]Gupta, R.C.,Gupta, P.I. and Gupta, R.D. (1998). Modeling failure time data by Lehmann alternatives, Communications in Statistics-Theory and Methods, 27, 887-904.

[15]Gupta, R.D. and Kundu, D. (2001). Exponentiated exponential family:An alternative to gamma and Weibull distributions, Biometrical Journal, 43, 117-130.

[16] Gurvich, M.R., DiBenedetto, A.T. and Ranade, S.V. (1997). A new statistical distribution for characterizing the random strength of brittle materials. Journal of Material Science, Vol.32, Issue 10, 2559-2564.

[17]Jones, M.C. (2004). Families of distributions arising from distributions of order statistics. Test, 13, 1-43.

[18]Korkmaz, M. Ç. and Genç, A. I. (2015). A Lifetime Distribution based on a transformation of a Two-Sided Power Variate. Journal of Statistical Theory and Applications, Vol. 14, No.3, 265-280.

[19]Korkmaz, M. Ç. and Genç, A. I. (2016). A New Generalized Two-sided Class of Distributions with Emphasis on Two-sided Generalized Normal Distribution. 
Communications in Statistics-Simulation and Computation, DOI: 10.1080/03610918.2015.1005233.

[20]Kumar, D. (2016). Ratio and Inverse Moments of Marshall-Olkin extended Burr type XII Distribution based on Lower Generalized Order-Statistics. Journal of Data Sciences. 14(1), 53-66.

[21]Maiti, S. S. and Pramanik, S. (2015). Odds Generalized Exponential-Exponential Distribution. Journal of Data Sciences. 13(2). 733-754.

[22] Marshall, A.W. and Olkin, I. (1997). A new method for adding a parameter to a family of distributions with application to the exponential and Weibull families. Biometrika, 84, 3, 641-652.

[23] Mielke, P.W. (1973). Another family of distributions for describing and analyzing precipitation data. Journal of Applied Meteorology,12, 275-280.

[24] Nadarajah, S. and Kotz, S. (2006). The exponentiated type distributions, Acta Applicandae Mathematica, 92, 97-111.

[25]Nadarajah, S. and Kotz, S. (2007). On the alternative to the Weibull function. Engineering Fracture Mechanics, Vol. 74, issue 3, 451-456.

[26] Nascimento, A.D.C., Bourguignon, M., Zea, L.M., Santos-Neto, M.S., Silva, R.B. and Cordeiro, G.M. (2014). The Gamma Extended Weibull family of distributions. Journal of Statistical Theory and Applications, Vol. 13, No.1, 1-16.

[27]Ristic, M. M. and Balakrishnan, N. (2012). The gamma-exponentiated exponential distribution. Journal of Statistical Computation and Simulation, 82, 1191-1206.

[28] Santos-Neto, M.S., Bourguignon, M., Zea, L.M., Nascimento, A.D.C., and Cordeiro, G.M. (2014). The Marshall-Olkin extended Weibull family of distributions .Journal of Statistical Distributions and Applications, 1:9, DOI:10.1186/2195-5832-1-9.

[29] Shao, Q., Chen, Y. D. and Zhang, L. (2008). An extension of three-parameter Burr III distribution for low-flow frequency analysis. Computational Statistics and Data Analysis, $52,1304-1314$.

[30] Smith, R.L. and Naylor, J.C. (1987). A comparison of Maximum Likelihood and Baysian Estimators for the Three-Parameter Weibull Distribution. Journal of the Royal Statistical Society. Series C (Applied Statistics), Vol.36, No.3 , 358-369.

[31]Tahir, M.H., Zubair, M., Mansoor, M., Cordeiro, G.M., Alizadeh, M. and Hamedani, G.G. (2015a). A new Weibull-G Family of Distributions. Hacettepe Journal of Mathematics and Statistics (to appear).

[32]Tahir, M.H., Cordeiro, G.M., Alizadeh, M., Mansoor, M., Zubair, M. and Hamedani, G.G. (2015b). The odd generalized exponential family of distributions with applications. Journal of Statistical Distributions and Applications, DOI 10.1186/s40488-014-0024-2.

[33] Yousof, H.M., Afify, A.Z., Hamidani, G.G. and Aryal, G.(2016). The Burr X Generator of Distributions for Lifetime Data. Journal of Statistical Theory and Applications, Vol.16. 119.

[34]Zografos, K. and Balakrishnan, N. (2009). On families of beta- and generalized gammagenerated distributions and associated inference. Statistical Methodology,6 344-362. 


\author{
Shahzadi Arifa \\ Department of Social Sciences \\ National College of Business Administration and Economics \\ Lahore, Pakistan \\ shahzadiarifa@gmail.com \\ Mohammad Zafar Yab \\ Department of Social Sciences \\ National College of Business Administration and Economics \\ Lahore, Pakistan \\ drzafaryab@yahoo.com \\ Azeem Ali \\ Department of Social Sciences \\ National College of Business Administration and Economics \\ Lahore, Pakistan \\ syedazeemali@gmail.com
}

